\title{
HOPF INVARIANTS FOR REDUCED PRODUCTS OF SPHERES
}

\author{
HANS JOACHIM BAUES
}

\begin{abstract}
Let $S_{m}^{n}$ be the $m$ th reduced product complex of the even dimensional sphere $S^{n}$. Using 'cup'-products, James defined a Hopf invariant homomorphism

$$
H_{m}^{n}: \pi_{m n-1}\left(S_{m-1}^{n}\right) \rightarrow \mathbf{Z}
$$

such that $H_{2}^{n}$ is the classical Hopf invariant. Extending the result of Adams on $H_{2}^{n}$ we determine the image of $H_{m}^{n}$. Partial calculations were made by Hardie and Shar.
\end{abstract}

1. Hopf invariants and higher order Whitehead products. In [9] James proved, that the reduced product complex $S_{\infty}^{n}$ of the sphere $S^{n}$ is homotopy equivalent to the loop space $\Omega S^{n+1}$. The complex $S_{\infty}^{n}$ has a natural $C W$ decomposition

$$
S_{\infty}^{n}=S^{n} \cup e^{2 n} \cup \ldots \cup e^{m n} \cup \ldots
$$

the $m n$ skeleton of which is denoted by $S_{m}^{n}, S_{1}^{n}=S^{n}$. In this paper we will be concerned with the homomorphism

$$
H_{m}^{n}: \pi_{m n-1}\left(S_{m-1}^{n}\right) \rightarrow \mathbf{Z}
$$

where $n$ is even and $m \geqslant 2$. This homomorphism is a generalization due to James [10] of Steenrod's definition of the Hopf invariant [21]: Let $\alpha \in$ $\pi_{m n-1}\left(S_{m-1}^{n}\right)$; one can choose generators $a_{1}, a_{m-1}$ and $x$ of dimension $n$, $(m-1) n$ and $m n$ respectively in the integral cohomology of the complex $S_{m-1}^{n} \cup_{\alpha} E^{m n}$. Then the Hopf invariant $H_{m}^{n}(\alpha)$ is defined to be the integer for which $a_{1} \cup a_{m-1}=H_{m}^{n}(\alpha) x . H_{2}^{n}$ is the classical Hopf invariant [8].

Let the element $\left[i_{n}\right]^{m} \in \pi_{m n-1}\left(S_{m-1}^{n}\right)$ be given by an attaching map of the cell $e^{m n}$ in $S_{m}^{n}$. This element is an $m$ th order Whitehead product [16], [6]. For example $\left[i_{n}\right]^{2}$ is the Whitehead product $\left[i_{n}, i_{n}\right]$ of a generator $i_{n} \in \pi_{n}\left(S^{n}\right)$. It is well known that $H_{2}^{n}\left(\left[i_{n}, i_{n}\right]\right)=2$ and more generally $H_{m}^{n}\left(\left[i_{n}\right]^{m}\right)=m$; see $\S 3$.

On the other hand we have $H_{2}^{n}\left(\sigma_{n}\right)=1$ for the Hopf elements $\sigma_{n} \in$ $\pi_{2 n-1}\left(S^{n}\right), n=2,4,8$ [8] and im $H_{2}^{n}=2 \mathrm{Z}$ if $n \neq 2,4,8$ by the celebrated theorem of Adams on Hopf invariants [2]. Moreover Toda showed in [23, p. 175] that for a prime number $p$, there exist $\alpha_{p} \in \pi_{2 p-1}\left(S_{p-1}^{2}\right)$ (and $\alpha_{2}=\sigma_{2}$ if $p=2$ ) such that $H_{p}^{2}\left(\alpha_{p}\right)=1$. We will prove that the elements $\alpha_{p}$ and the Hopf elements $\sigma_{2}, \sigma_{4}, \sigma_{8}$ are the only elements of Hopf invariant one.

TheOREM A.

Received by the editors June 2, 1975.

AMS (MOS) subject classifications (1970). Primary 55E25.

Key words and phrases. Hopf invariant, Whitehead product, reduced product space. 


$$
\operatorname{im} H_{m}^{n}= \begin{cases}\mathbf{Z}, & \text { if } m=2 \text { and } n=2,4,8 \\ \mathbf{Z}, & \text { if } n=2 \text { and } m \text { a prime number } \\ m \mathbf{Z}, & \text { otherwise. }\end{cases}
$$

ACKNOWLEDGEMENT. It follows easily from (1.3), (c), (ii) of [6] that im $H_{m}^{n}$ $=m \mathbf{Z}$ if $n \geqslant 4$ and $m$ is an odd prime. Using this result Shar showed that im $H_{m}^{n}=m \mathbf{Z}$ for $n \geqslant 4$ and $m \geqslant 3$ [19], [20]. Thus we only have to prove im $H_{m}^{2}=m \mathbf{Z}$ in case $m$ is not a prime. Using our method of proof one can also deduce Shar's result. Our proof is nevertheless different from Shar's proof, since in the case of the reduced product of a 2-sphere we cannot use Lemma 2.1 in [19].

From the exact sequence of the pair $\left(S_{\infty}^{n}, S_{m-1}^{n}\right)$ we have a short exact sequence:

$$
0 \rightarrow \pi_{m n}\left(S_{\infty}^{n}, S_{m-1}^{n}\right) \stackrel{\partial}{\rightarrow} \pi_{m n-1}\left(S_{m-1}^{n}\right) \stackrel{\iota}{\rightarrow} \pi_{m n}\left(S^{n+1}\right) \rightarrow 0 .
$$

There is a generator $\omega_{m}^{n}$ of $\pi_{m n}\left(S_{\infty}^{n}, S_{m-1}^{n}\right) \cong \mathbf{Z}$ with $\partial \omega_{m}^{n}=\left[i_{n}\right]^{m}$. Therefore $\iota(\alpha)$ is a nontrivial element if $0<H_{m}^{n}(\alpha)<m$. For example $\iota\left(\alpha_{p}\right)$ is the well-known element of order $p$ in $\pi_{2 p}\left(S^{3}\right)$ and $\iota\left(\sigma_{n}\right)$ is the nontrivial suspension of the Hopf element. By Theorem A, this method will not yield further nontrivial elements in the homotopy groups of spheres. On the other hand the short exact sequence above immediately implies the following corollary of Theorem A:

(1.2) Corollary. In case im $H_{m}^{n}=m \mathbf{Z}$, we have $\pi_{m n-1}\left(S_{m-1}^{n}\right)=\mathbf{Z} \oplus$ $\pi_{m n}\left(S^{n+1}\right)$ and $\left[i_{n}\right]^{m}$ generates the infinite cyclic summand.

Using this corollary we will determine the order of the Whitehead products $\left[i_{n},\left[i_{n}\right]^{m-1}\right] \in \pi_{m n-2}\left(S_{m-2}^{n}\right)$, where $i_{n} \in \pi_{n}\left(S_{m-2}^{n}\right)$ is a generator and $m>2$. That $m\left[i_{n},\left[i_{n}\right]^{m-1}\right]=0$, is a special case of the Jacobi identity for the higher order Whitehead products (Hardie [5], [6]). Moreover Hardie has proven in [6] that $\left[i_{2},\left[i_{2}\right]^{m-1}\right]=0$ if $m$ is a prime. In fact:

(1.3) COROLlaRY. $\left[i_{n},\left[i_{n}\right]^{m-1}\right]=0$ if and only if $n=2$ and $m$ is a prime; and $\left[i_{n},\left[i_{n}\right]^{m-1}\right]$ is an element of order $m$ otherwise.

If $m=3$ this is a well-known result on the iterated Whitehead product $\left[i_{n}\right.$, $\left.\left[i_{n}, i_{n}\right]\right] \in \pi_{3 n-2}\left(S^{n}\right)$; cf. [15], [13]. Shar has proven the result of Corollaries (1.2) and (1.3) in case $n \geqslant 4$ [19]. Compare also Hardie's result (0.2)(b) in [7].

Proof of (1.3). We consider the exact sequence

$$
\pi_{m n-1}\left(S_{m-1}^{n}\right) \stackrel{j}{\rightarrow} \pi_{m n-1}\left(S_{m-1}^{n}, S_{m-2}^{n}\right) \stackrel{\partial}{\rightarrow} \pi_{m n-2}\left(S_{m-2}^{n}\right) .
$$

Using the Nakaoka Toda formula [5], [6] we have $j\left(\left[i_{n}\right]^{m}\right)=m\left[i_{n}, \omega_{m-1}^{n}\right]$. The relative Whitehead product $\left[i_{n}, \omega_{m-1}^{n}\right]$ generates the infinite cyclic part of $\pi_{m n-1}\left(S_{m-1}^{n}, S_{m-2}^{n}\right)$, see 1.4 ,p. 262 in [11]. Since $\partial\left[i_{n}, \omega_{m-1}^{n}\right]= \pm\left[i_{n},\left[i_{n}\right]^{m-1}\right]$, the result follows using (1.2).

2. The chain algebra of $\Omega S_{\infty}^{n}$. We obtain our result on the Hopf invariant $H_{m}^{n}$ by examining $H_{m n-2}\left(\Omega S_{m-1}^{n}\right)$, with integral coefficients. Let the homomorphism

$$
\tau: \pi_{m n-1}\left(S_{m-1}^{n}\right) \cong \pi_{m n-2}\left(\Omega S_{m-1}^{n}\right) \rightarrow H_{m n-2}\left(\Omega S_{m-1}^{n}\right)
$$


be given by composition with the Hurewicz homomorphism as in [17]. We will prove:

THEOREM B. There is a homomorphism $T: H_{m n-2}\left(\Omega S_{m-1}^{n}\right) \rightarrow \mathbf{Z}$ such that

$$
\operatorname{T\tau }\left(\left[i_{n}\right]^{m}\right)= \begin{cases}p, & \text { if } m=p^{v} \text { is a power of a prime } p, \\ 1, & \text { otherwise. }\end{cases}
$$

(2.1) COROLlaRY. If $m$ is not a power of a prime number, then im $H_{m}^{n}=m \mathbf{Z}$. If $m=p^{v}$ is a power of the prime $p$, then im $H_{m}^{n}=m \mathbf{Z}$ or im $H_{m}^{n}=p^{v-1} \mathbf{Z}$.

Proof of (2.1). Let $\alpha \in \pi_{m n-1}\left(S_{m-1}^{n}\right)$ be given such that im $H_{m}^{n}=H_{m}^{n}(\alpha) \mathbf{Z}$. By use of the exact sequence (1.1) we obtain $\pi_{m n-1}\left(S_{m-1}^{n}\right) \cong \mathbf{Z} \oplus T$ ( $T$ a torsion group) and $\alpha$ generates the infinite cyclic summand. Since $\left[i_{n}\right]^{m}$ $=k \alpha+t(t \in T)$ we have $m=k H_{m}^{n}(\alpha)$. On the other hand $k$ is a divisor of $T \tau\left(\left[i_{n}\right]^{m}\right)$, and so the corollary follows from Theorem B.

For the proof of Theorem B we need the chain algebra of $\Omega S_{\infty}^{n}$ in the sense of [1]. Let $A=A\left(S_{\infty}^{n}\right)=A\left[x_{1}, x_{2}, \ldots\right]$ be the unitary ring, which is freely generated by the elements $x_{1}, x_{2}, \ldots$ and graded by $\operatorname{deg}\left(x_{i}\right)=n i-1$. We define a differential $d: A \rightarrow A$ on generators by

$$
d x_{i}=\sum_{j=1}^{i-1}\left(\begin{array}{l}
i \\
j
\end{array}\right) x_{j} x_{i-j} .
$$

For products $a b$ in $A$ we define $d(a b)=(d a) b+(-1)^{k} a(d b)$, where $k=$ $\operatorname{deg}(a)$. Let $A^{m}=A\left[x_{1}, \ldots, x_{m}\right]$ be the chain subalgebra of $A$ generated by $x_{1}, \ldots, x_{m}$. Then we obtain from [3] and [14]:

(2.3) Lemma. There exists an isomorphism $\Theta: H_{*}\left(A^{m-1}, d\right) \rightarrow H_{*}\left(\Omega S_{m-1}^{n}\right)$, such that $\Theta\left\{d x_{m}\right\}=\tau\left(\left[i_{n}\right]^{m}\right)$. Here $d x_{m}$ is a cycle in $A^{m-1}$ representing the nonzero homology class $\left\{d x_{m}\right\}$.

(2.4) Proof of Theorem B. Let $C_{k}$ be the $k$ th chain group of the chain complex $A^{m-1}, k \geqslant 0$. Let $X_{i} \subset C_{n m-i}$ be generated by monomials in $A^{m-1}$ with at most $i$ factors, $i=1,2,3$. Let $Y_{i} \subset C_{n m-i}$ be generated by monomials in $A^{m-1}$ with more than $i$ factors. By (2.2) we obtain restrictions $d_{j}^{\prime}$ and $d_{j}^{\prime \prime}$ $(j=1,2)$ of $d$ such that the following diagram commutes:

$$
\begin{array}{llll}
C_{n m-1} & = & X_{1} \oplus \quad Y_{1} \\
d \downarrow & & & \downarrow d_{1}^{\prime} \oplus d_{2}^{\prime} \\
C_{n m-2} & = & X_{2} \oplus \quad Y_{2} \\
d \downarrow & & & \downarrow d_{1}^{\prime \prime} \oplus d_{2}^{\prime \prime} \\
C_{n m-3} & = & X_{3} \oplus Y_{3}
\end{array}
$$

Since $X_{1}=0$ we obtain by (2.3) the isomorphism

$$
\Theta^{-1}: H_{n m-2}\left(\Omega S_{m-1}^{n}\right) \rightarrow \operatorname{ker} d_{1}^{\prime \prime} \oplus \operatorname{ker} d_{2}^{\prime \prime} / \operatorname{im} d_{2}^{\prime} \text {. }
$$

A simple computation using (2.2) shows that ker $d_{1}^{\prime \prime} \cong \mathbf{Z}$ and is generated by $d x_{m} / g_{m}$, where $g_{m}=\operatorname{gcd}\left\{\left(\begin{array}{c}m \\ i\end{array}\right) \mid 0<i<m\right\}$. Let $T$ be given by composition of $\Theta^{-1}$ with projection on ker $d_{1}^{\prime \prime}$. Then we have $T \tau\left(\left[i_{n}\right]^{m}\right)=g_{m}$ by (2.3). One can check that $g_{m}=p$ if $m=p^{v}$ is a power of the prime $p$ and that $g_{m}=1$ otherwise. This completes the proof of Theorem B. 
3. Proof of Theorem A. Let $\alpha \in \pi_{m n-1}\left(S_{m-1}^{n}\right)$ and let the complex $K$ $=S_{m-1}^{n} \cup_{\alpha} E^{m n}$ be obtained by attaching an oriented $m n$ cell $E^{m n}$ to $S_{m-1}^{n}$ by a map $f$ of homotopy class $\alpha$. Using the result of Serre on the cohomology of $S_{\infty}^{n}$, we can choose generators $a_{i} \in H^{n i}(K)$, where $1 \leqslant i<m$, such that

$$
a_{1}^{i}=i ! a_{i}
$$

cf. Theorem 18 on p. 488 in [18]. Let the Hopf invariant $H_{m}^{n}(\alpha)$ be defined by use of these generators. In case $\alpha=\left[i_{n}\right]^{m}$ and $K=S_{m}^{n}$ this implies $H_{m}^{n}\left(\left[i_{n}\right]^{m}\right)$ $=m$.

For the proof of Theorem A we need only show that the assumption im $H_{m}^{2}=p^{v-1} \mathbf{Z}$ leads to a contradiction if $m=p^{v}$ is a prime power with $v>1$; see (2.1). Assuming this, it follows that there exists an element $\alpha$ such that $H_{m}^{2}(\alpha)=p^{v-1}$. Thus in $K$ we have

$$
a_{1} \cup a_{m-1}=p^{v-1} x .
$$

Multiplying by $(m-1)$ !, we obtain from (1):

$$
a_{1}^{m}=a_{1} \cup(m-1) ! a_{m-1}=\left(p^{v}-1\right) ! p^{v-1} x=p^{v} ! p^{-1} x .
$$

Thus if $q=p^{r-1}$, we have $q p=m$, and

$$
a_{1}^{m}=\left(a_{1}^{q}\right)^{p}=\left(q ! a_{q}\right)^{p}=(q !)^{p} a_{q}^{p} .
$$

Therefore we obtain $a_{q}^{p}=r_{p} x$, where

$$
r_{p}=\left(p^{v-1} !\right)^{-p}\left(p^{v} !\right) p^{-1} \text {. }
$$

Let $a_{q, p} \in H^{2 q}\left(K, \mathbf{Z}_{p}\right)$ and $x_{p} \in H^{2 m}\left(K, \mathbf{Z}_{p}\right)$ be generators, which correspond to $a_{q}$ and $x$ in case of integral coefficients. Since $r_{p} \neq 0 \bmod p$, we have:

$$
\left(a_{q, p}\right)^{p}=r_{p} x_{p} \neq 0 .
$$

The following arguments generalize the method of Nakaoka and Toda on p. 12 in [15] and the method of Hardie on p. 247 in [6]: Since the suspension $\Sigma$ of a higher order Whitehead product is trivial, there exists a mapping $\rho$ : $\sum S_{m-1}^{2} \rightarrow S^{2 q+1}$ which induces an isomorphism of cohomology in dimension $2 q+1$. Let $g=\rho \circ(\Sigma f)$, where $f \in \alpha$. Then we have an extension $\bar{\rho}$ : $\Sigma K \rightarrow S^{2 q+1} \cup_{g} E^{2 m+1}=K_{g}$ such that $\bar{\rho}$ induces isomorphism of cohomology in dimension $2 q+1$ and $2 m+1$.

We examine the cases $p$ odd and $p=2$ separately. If $p$ is odd, it follows from (6) that the Steenrod $p$ th power

$$
P^{q}\left(a_{q, p}\right)=\left(a_{q, p}\right)^{p} \neq 0 .
$$

By the naturality of the reduced power operation (using $\bar{\rho}$ ) and the fact that they commute with suspension, we obtain from (7) an isomorphism:

$$
P^{q}: H^{2 q+1}\left(K_{g}, \mathbf{Z}_{p}\right) \rightarrow H^{2 m+1}\left(K_{g}, \mathbf{Z}_{p}\right) .
$$

Hence the $\bmod p$ Hopf invariant of $g$ is nontrivial. Now by Theorem 5 of [13] this is only the case if $q=2$ and so we have the required contradiction.

On the other hand if $p=2$, it follows from (6) that the Steenrod square

$$
\operatorname{Sq}^{2 q}\left(a_{q, 2}\right)=\left(a_{q, 2}\right)^{2}=r_{2} x_{2} \neq 0 \text {. }
$$


Since the suspension $\Sigma_{*}: \pi_{2 m-1}\left(S^{2 q}\right) \rightarrow \pi_{2 m}\left(S^{2 q+1}\right)$ is an epimorphism by the Freudenthal theorem, we have a mapping $\bar{g}: S^{2 m-1} \rightarrow S^{2 q}$ such that $\Sigma \bar{g}$ and $g$ are homotopic. We also obtain a homotopy equivalence $t: \Sigma K_{\bar{g}} \rightarrow K_{g}$. By the naturality of the Steenrod squares (using $\bar{\rho}$ and $t$ ) and the fact that they commute with suspension we obtain from (9) an isomorphism:

$$
\mathrm{Sq}^{2 q}: H^{2 q}\left(K_{\bar{g}}, \mathbf{Z}_{2}\right) \rightarrow H^{2 m}\left(K_{\bar{g}}, \mathbf{Z}_{2}\right) .
$$

Hence the Hopf invariant $H_{2}^{2 q}(\bar{g})$ is odd. Since this is only the case if $m=2$, 4,8 we have the required contradiction for $m=2^{v}$ and $v>3$; cf. 4.5 in [22] and [2]. Thus we still have to find a contradiction in case $m=4$ and $m=8$.

In case $m=4$ we assume that there exists an $\alpha$ such that $H_{4}^{2}(\alpha)=2$. By use of the exact sequence (1.1), $\iota(\alpha) \in \pi_{8}\left(S^{3}\right)$ is a nontrivial element. Using the exact sequence in the proof of (1.3), we know that $j(\alpha)$ is nontrivial and thus $\iota(\alpha)$ is an element of filtration 3. Since James has proven that all elements of $\pi_{8}\left(S^{3}\right)$ have filtration less than 3, we have a contradiction; cf. row 3 , p. 309 in [12].

In case $m=8$ we consider the following diagram:

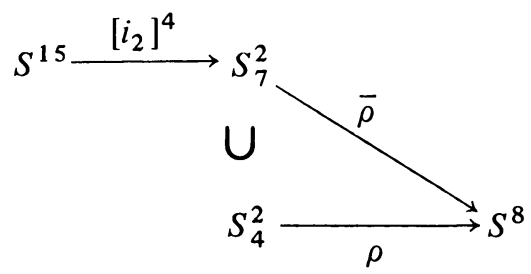

where $\rho$ is a mapping which induces an isomorphism of homology in dimension 8 .

In [4] we prove that there exists an extension $\bar{\rho}$ of $\rho$ such that

$$
\bar{\rho}_{*}\left(\left[i_{2}\right]^{4}\right)=35\left[i_{8}, i_{8}\right] \in \pi_{15}\left(S^{8}\right)
$$

If we assume that $\operatorname{im} H_{8}^{2}=4 \mathrm{Z}$, then there exists an $\alpha \in \pi_{15}\left(S_{7}^{2}\right)$ such that $H_{8}^{2}(\alpha)=4$ and such that $\left[i_{2}\right]^{4}=2 \alpha+t$; compare the proof of $(2.1)$. Since $\pi_{16}\left(S^{3}\right)=\mathbf{Z}_{6}$, the element $t$ has order at most 3 , using the exact sequence (1.1). From Toda's book [23] we know that $\pi_{15}\left(S^{8}\right)=\mathbf{Z}+\mathbf{Z}_{120}$, and the Hopf element $\sigma_{8}$ generates the infinite cyclic summand. Let $\sigma$ be the generator of $\mathbf{Z}_{120}$. Then we have $\left[i_{8}, i_{8}\right]= \pm\left(2 \sigma_{8}-15 \sigma\right)$; see 5.16 on p. 50 in [23]. Now there exist $w, a, b \in \mathbf{Z}$ such that $\bar{\rho}_{*}(t)=40 \cdot w \sigma$ and $\bar{\rho}_{*}(\alpha)=a \sigma_{8}+b \sigma$. Thus we obtain from (11):

$$
\pm 35\left(2 \sigma_{8}-15 \sigma\right)=2\left(a \sigma_{8}+b \sigma\right)+40 \cdot w \sigma .
$$

Since $35 \cdot 15 \not \geq 2 b+40 w \bmod 120$ for $b, w \in \mathbf{Z}$, we have the required contradiction and the proof of Theorem $\mathrm{A}$ is complete.

As pointed out by the referee in case $m=8$ a filtration argument is as well available, since the reduced product filtration of the generator of $\pi_{16}\left(S^{3}, 2\right)$ is 2. 


\section{REFERENCES}

1. J. F. Adams and P. J. Hilton, On the chain algebra of a loop space, Comment. Math. Helv. 30 (1956), 305-330. MR 17, 1119.

2. J. F. Adams, On the non-existence of elements of Hopf invariant one, Ann. of Math. (2) 72 (1960), 20-104. MR 25 \#4530.

3. H. J. Baues, Der Pontryagin-Ring von Quotienten eines Torus, Math. Z. 134 (1973), 221-228. MR 48 \# 12520.

4. Hindernisse in dem Produkt von Suspensionen, Math. Ann. 200 (1973), 11-23. MR $49 \# 8011$.

5. K. A. Hardie, A proof of the Nakaoka-Toda formula, Pacific J. Math. 14 (1964), 1249-1254. MR 30 \#2507.

6. _ Higher Whitehead products, Quart. J. Math. Oxford Ser. (2) 12 (1961), 241-249. MR 25 \#4521.

7. \# 4057.

8. H. Hopf, Über die Abbilungen von Sphären auf Sphären niedrigerer Dimension, Fund. Math. 25 (1935), 427-440.

9. I. M. James, Reduced product spaces, Ann. of Math. (2) 62 (1955), 170-197. MR 17, 396.

10. _ Note on cup-products, Proc. Amer. Math. Soc. 8 (1957), 374-383. MR 19, 974.

11. On the homotopy groups of certain pairs and triads, Quart. J. Math. Oxford Ser. (2) 5 (1954), 260-270. MR 16, 948.

12. _ Filtration of the homotopy groups of spheres, Quart. J. Math. Oxford Ser. (2) 9 (1958), 301-309. MR 20 \#266.

13. A. Liulevicius, The factorization of cyclic reduced powers by secondary cohomology operations, Proc. Nat. Acad. Sci. U.S.A. 46 (1960), 978-981. MR 24 \#2383.

14. R. J. Milgram, Iterated loop spaces, Ann. of Math. (2) 84 (1966), 386-403. MR 34 \#6767.

15. M. Nakaoka and H. Toda, On Jacobi identity for Whitehead products, J. Inst. Polytech.

Osaka City Univ. Ser. A. 5 (1954), 1-13. MR 16, 505.

16. G. J. Porter, Higher-order Whitehead products, Topology 3 (1965), 123-135. MR 30 \#261.

17. H. Samelson, A connection between the Whitehead and the Pontryagin product, Amer. J. Math. 75 (1953), 744-752. MR 15, 731.

18. J.-P. Serre, Homologie singuliere des espaces fibrés. Applications, Ann. of Math. (2) 54 (1951), 425-505. MR 13, 574.

19. A. Shar, $\pi_{m n-2}\left(S_{m-2}^{n}\right)$ contains an element of order $m$, Proc. Amer. Math. Soc. 34 (1972), 303-306. MR 45 \#1166.

20. The homotopy groups of spaces whose cohomology is a $Z_{p}$ truncated polynomial algebra, Proc. Amer. Math. Soc. 38 (1973), 172-178. MR 46 \#9975.

21. N. E. Steenrod, Cohomology invariants of mappings, Ann. of Math. (2) 50 (1949), 954-988. MR 11, 122.

22. Cohomology operations (Lectures by N. E. Steenrod and revised by D. B. A. Epstein), Ann. of Math. Studies, no. 50, Princeton Univ. Press, Princeton, N. J., 1962. MR 26 \# 3056 .

23. H. Toda, Composition methods in homotopy groups of spheres, Ann. of Math. Studies, no. 49, Princeton Univ. Press, Princeton, N. J., 1962. MR 26 \#77.

Sonderforschungsbereich 40 'Theoretische Mathematik', Mathematisches Institut Der Universität, D-5300 Bonn, Wegelerstr. 10, Federal Republic of Germany 